\title{
La educación a través del arte: de la teoría a la realidad del sistema educativo
}

\author{
Silvia VIÑAO MANZANERA \\ Universidad Católica de Murcia (UCAM) \\ silviavinao@gmail.com
}

Recibido: $14 / 07 / 2012$

Aceptado: 28/09/2012

\section{Resumen}

El arte es una importante herramienta pedagógica que encierra múltiples posibilidades. Numerosos teóricos, defensores de la educación a través del arte, así lo afirman. En este artículo trataremos de hacer una breve revisión de dichas teorías, para después realizar un sondeo sobre la incidencia del arte en la Educación Secundaria Obligatoria y el Bachillerato, comprobando de este modo, la relación entre la teoría y la práctica educativa.

Palabras clave: Educación, Arte, Educación secundaria.

The education through art:

from the theory to the reality of the educative system

\begin{abstract}
Art is a relevant educational tool involving many possibilities. This is stated by several theorists defending education through art. In this article we intend to provide a brief survey of the above theories, as well as investigate the presence of arts education in the Compulsory Secondary Education and the "Bachillerato", thus checking the relationship existing between theory and educational practice.
\end{abstract}

Keywords: Education, Art, Secondary Education.

\section{Referencia normalizada}

VIÑAO MANZANERA, Silvia (2012): "La educación a través del arte: de la teoría a la realidad del sistema educativo". Estudios sobre el mensaje periodístico. Vol. 18, núm. especial noviembre, págs.: 919927. Madrid, Servicio de Publicaciones de la Universidad Complutense.

Sumario: 1. Introducción. 2. Metodología. 3. Consideraciones sobre la educación a través del arte. 3.1. La contemplación de la belleza. 3.2. H. Gardner y el desarrollo holístico del ser humano. 4. La incidencia del arte en la Educación Secundaria. 4.1. La realidad de la asignatura de Educación Plástica y Visual en la ESO. 4.2. Resultados de las encuestas. 5. Conclusiones. 6. Referencias bibliograficas.

\section{Introducción}

Esta investigación tiene como origen dos campos relacionados aunque diferentes. Por un lado, la revisión de las teorías sobre el valor pedagógico del arte y por otro, la incidencia real del arte en el sistema educativo actual.

Con el fin de destacar el valor del arte en la educación nos basaremos en la corriente pedagógica que defiende la educación a través del arte. En este sentido, destacaremos las teorías postuladas por Gardner y su defensa de un desarrollo holístico de las capacidades humanas, así como de la concepción pedagógica que propone el arte como una excelente herramienta para desarrollar capacidades tales como la creatividad, la intuición, la espontaneidad, la imaginación o las de índole manuales.

La fundamentación de la indagación realizada se basa en la disparidad existente entre las teorías anteriormente mencionadas y la realidad del sistema educativo actual, hecho que demostraremos en la parte practica de esta investigación. Para anali- 
zar esta situación me basare en la propia experiencia personal como profesora de educación plástica en diferentes institutos públicos y colegios privados durante cuatro años, y en encuestas realizadas a alumnos de Educación Secundaria Obligatoria (ESO) y Bachillerato de la región de Murcia.

\section{Metodología}

En la primera parte del artículo se expone la fundamentación teórica, de índole cualitativa, que sustenta la segunda parte en la que se analizan los resultados de una encuesta realizada a cien alumnos de la ESO y Bachillerato. Se combinan, pues, la metodología cualitativa con la cuantitativa.

\section{Consideraciones sobre la educación a través del arte}

Actualmente la mayoría de los pedagogos, psicólogos e investigadores que han ahondado en este tema defienden los beneficios del arte como agente enriquecedor para el ser humano. En esta línea, podemos mencionara estudiosos como H. Read, E. W. Eisner o H. Gardner, entre otros, que destacan las potencialidades del arte como herramienta educativa. Este concepto del arte unido a una preocupación por el desarrollo completo del ser humano, nos lleva defender la educación a través del arte.

En la educación a través del arte lo importante ya no es el resultado final, sino la transformación que la práctica creativa o contemplativa es capaz de causar en el ser humano. Este punto de vista enriquece la práctica artística, que ya no es solo colorear o conseguir un resultado parecido a un modelo dado. No obstante, para ello hay que estar dispuestos a indagar en este nuevo enfoque y a valorar los aspectos cualitativos del arte (Eisner: 1998).

Para entender mejor este enfoque pasaremos a analizar algunos de los aspectos de las teorías más importantes al respecto:

\subsection{La contemplación de la belleza.}

Autores como H. Read valoran la contemplación de la belleza estética como una practica transformadora y por tanto formadora, ya que transmuta nuestro estado de ánimo en un sentido positivo:

"La contemplación de la belleza sustrae la sensibilidad de la presión de la vida, del propósito intencional del símbolo hacia un estado de animación suspendida, hacia un estado de serenidad" (Read, $\left.{ }^{3} 1975: 38\right)$.

Cuando la belleza no es sólo una imagen estéticamente correcta, sino que transmite una emoción; es decir, cuando provoca un estado de ánimo, estamos hablando de algo más que de meras cuestiones estéticas. El estado de ánimo que provoca la belleza puede llegar a ser un estado en el que la mente se abstrae por unos momentos de su discurso ordinario. 
Estamos hablando, pues, de un poder que, aunque subjetivo y difícil de cuantificar, no es por ello inexistente. Hablamos de un poder que la belleza tiene sobre la psique humana, lo mismo que podríamos hablar del poder de la música, pues tanto una como la otra tienen la capacidad de transmitir una sensación de relajación o plenitud. Este poder ha sido empleado en la actualidad en numerosos cursos de Arteterapia, y podría ser empleado en las aulas como medio para el desarrollo de la inteligencia emocional.

\subsection{H. Gardner y el desarrollo holístico del ser humano}

Otro aspecto fundamental en esta investigación es que el arte o la enseñanza del mismo, entendida de manera holística, no sólo desarrolla capacidades estéticas sino también creativas, intuitivas, imaginativas o expresivas, además de proporcionar un mayor autoconocimiento y otro modo de relacionarnos con el entorno. Capacidades todas ellas importantes en el desarrollo humano, como apunta Gardner, que nos acercan a un ideal de ser humano más consciente y más completo.

Gardner defiende el tipo de pensamiento creativo e intuitivo desarrollado a través del arte porque en nuestra sociedad se tiende, según él y otros autores, a privilegiar el pensamiento lógico racional. Sin embargo, Gardner no excluye el pensamiento lógico racional. Al contrario, propone una visión integradora de la valoración de los dos tipos de pensamiento. En palabras del propio Gardner, en su libro Educación artística y desarrollo humano,

"Sería tan erróneo afirmar que el desarrollo debe estudiarse exclusivamente desde la perspectiva del artista, como lo es sostener que sólo vale la pena tomar en serio la competencia científica final. Sin duda, una ciencia comprensiva del desarrollo humano necesita en cierto modo considerar el espectro completo de las capacidades y los talentos mostrados por los seres humanos maduros en diversas culturas" (Gardner, 1994: 26).

Lo que Gardner no comparte, al igual que otros autores, es el punto de vista que privilegia la lógica racional por encima de todo:

"Un grupo de filósofos con un marcado interés por las artes, especialmente el filósofo alemán Ernst Cassirer [...] y los filósofos norteamericanos Susanne Langer [...] y Nelson Goodman [...] desafiaron frontalmente estas ideas. Cada uno de estos estudiosos de la simbolización señaló que el punto de vista que privilegiaba 'la lógica por encima de todo' era restrictivo en exceso. Como especie, los seres humanos somos capaces de un amplio número de competencias simbólicas, cuyo alcance se extiende más allá de la lógica y del lenguaje en su atuendo científico" (Gardner, 1994: 27).

Así pues, tanto para Gardner como para los demás investigadores que valoran el arte como una importante herramienta pedagógica, el problema radica en que las capacidades artísticas quedan relegadas a un segundo plano. Como es obvio este hecho supone un perjuicio en el desarrollo holístico del ser humano. 
Según las tesis de Gardner, los niños de edad inferior a siete u ocho años se encuentran en la llamada "edad de oro de la creatividad"; es decir, en un periodo durante el cual la mayoría de los niños hacen gala de sus habilidades creativas, imaginativas, expresivas y artístico-productivas. No obstante, en la mayoría de los casos esta creatividad se atrofia con los años.

En este sentido, la investigación de Gardner nos parece sumamente interesante, ya que mientras que el pensamiento lógico racional se va desarrollando con los años, el artístico en la mayoría de los casos se atrofia. ¿Por qué la mayoría de los seres humanos pierde unas facultades que ya tenía en la infancia? Este es el interrogante investigado por Gardner, llegando a la conclusión de que el niño pierde estas capacidades en el momento en que empieza a hacer caso de las correcciones de los adultos y que tanto el sistema educativo como la sociedad tienden a valorar más el pensamiento lógico racional que el artístico. Como consecuencia, la mayoría de los adultos, han perdido o atrofiado sus capacidades artesanales, artísticas, espontáneas, expresivas, imaginativas, intuitivas y creativas.

Para apoyar dicha conclusión Gardner realiza una revisión del conocimiento científico sobre el desarrollo humano en general desde una perspectiva artística, intentando integrar estos conocimientos con los referentes a las prácticas educativas en diferentes ámbitos del saber, así como con las distintas formas de conocimiento, con el fin de conseguir un desarrollo de las capacidades humanas más completo. En este sentido, Gardner encuentra en el arte, y sobre todo en la práctica artística, una herramienta ideal:

"Esta revisión atestiguaba el desafío con el que se enfrentan los estudiantes que intentan sintetizar varias formas de conocimiento que van desde las formas de comprensión sensoriomotices e intuitivas a las habilidades artesanales que pueden desarrollar hasta un exquisito nivel de maestría, y a los cuerpos notacionales y formales de conocimiento habitualmente acentuados en las escuelas" (Gardner, 1994: 87).

Así pues, Gardner considera que algunos tipos de conocimiento son "habitualmente acentuados en las escuelas". De acuerdo con ello, propone la enseñanza de la práctica artística para contrarrestar esta tendencia y conseguir, de este modo, un desarrollo humano más completo en todos los campos del conocimiento.

El ideal del desarrollo holístico del ser humano se completa con la formulación de la teoría de las inteligencias múltiples. Lo que Gardner nos propone es una visión pluralista de la mente humana, que no tiene una sola forma de conocer y comprender la realidad, sino muchas facetas cognitivas distintas. Partiendo de este enfoque, después de analizar las distintas capacidades y los distintos modos de cognición de la manera más amplia posible, y teniendo en cuenta que algunas capacidades son difícilmente cuantificables, formuló la teoría de las "inteligencias múltiples", según la cual podemos organizar las capacidades en siete tipos de inteligencias: lingüística, lógico-matemática, espacial, musical, corporal, interpersonal e intrapersonal (Gardner, 1995).

Los dos tipos de inteligencias que tradicionalmente se consideraban más impor- 
tantes y que eran computadas en la mayoría de los tests, evaluadas por los científicos y fomentadas en las escuelas, eran las dos primeras. Ahora bien ¿cuáles son para Gardner las más importantes?:

"Jean Piaget, el gran psicólogo evolutivo, pensaba que estaba estudiando toda la inteligencia, pero yo creo que lo que él estudiaba era el desarrollo de la inteligencia lógico-matemática. Pese a nombrar en primer lugar las inteligencias lingüística y lógico-matemática, no lo hago porque piense que son las más importantes: de hecho, estoy convencido de que las siete inteligencias tienen el mismo grado de importancia. En nuestra sociedad, sin embargo, hemos puesto las inteligencias lingüística y lógico-matemática, en sentido figurado, en un pedestal" (Gardner, 1995: 26).

Queda claro que Gardner defiende ciertos tipos de conocimiento, no por que los considere más importantes, sino porque tradicionalmente han estado relegados. Por tanto, una educación que tenga en cuenta el desarrollo de todas las capacidades humanas será más completa y contribuirá, junto a otros factores, a obtener como resultado un desarrollo humano más completo.

\section{La incidencia del arte en la Educación Secundaria.}

Una vez identificadas las potencialidades que encierra esta vinculación entre arte y educación, podríamos plantear como parte de esta investigación averiguar si las virtudes, anteriormente expuestas, del arte como herramienta educativa, se aprovechan en el sistema educativo actual, y más aún, si al arte se le da el valor real que pedagogos y psicólogos tan reconocidos como los anteriormente citados le confieren.

Para ello, hemos decidido centrarnos en el estudio de la situación en la ESO y el Bachillerato. En la ESO el alumnado se encuentran en una edad clave en la formación del individuo. Los alumnos ya tienen la suficiente madurez como para apreciar ciertos matices del arte que antes pasarían desapercibidos, pero aún está en una etapa de formación de la personalidad donde se están desarrollando sus capacidades y se están concretando sus gustos, sus aficiones e incluso sus preferencias profesionales. Es más, se trata de una etapa de cambios durante la cual el auto conocimiento y el conocimiento de su entorno cobran una especial relevancia. Así pues, en este periodo crucial de la formación de la persona, el arte puede ser una interesante herramienta y un excelente método de expresión de todo aquello que no se puede expresar con palabras.

\subsection{La realidad de la asignatura de Educación Plástica y Visual en la ESO}

Dado lo expuesto hasta ahora y teniendo en cuenta el auge actual de la imagen en los distintos medios de comunicación o entretenimiento, es de suponer que la Educación Plástica y Visual fuese una asignatura especialmente valorada en la ESO. Sin embargo, la realidad dista bastante de estos planteamientos. Según el Real Decreto 1631/2006 de 29 de Diciembre, publicado en el BOE del 5 de Enero de 2007, la Educación Plástica aparece entre las asignaturas a impartir en los tres primeros cursos de los cuatro de que consta la ESO, pero no tiene que impartirse en todos ellos. Es decir, 
puede impartirse en uno o dos cursos de la ESO, pero ya no es obligatoria en todos como ocurría anteriormente.

Como consecuencia de esta ley la asignatura de Educación Plástica ha visto reducida el número de horas lectivas, así como los cursos donde se impartía según establecían las comunidades autónomas. En la de Murcia, por ejemplo, además de reducir las horas lectivas, ha desaparecido de $2^{\circ}$ de la ESO, en $1^{\circ}$ y $3^{\circ}$ sigue siendo obligatoria y en $4^{\circ}$ optativa.

Durante mi etapa como profesora de Educación Plástica (años 1997-2000), pude comprobar como la asignatura de Educación Plástica era calificada a menudo, entre los alumnos, como una "Maria" y como éstos estaban totalmente desvinculados del arte.

En una ocasión, estando en clase con los alumnos de $2^{\circ}$ de la ESO les empecé a hablar de Van Gogh y no sabían quien era, pero si sabían quien era "La oreja de Van Gogh". Lejos de escandalizarme, aproveché la ocasión para despertar su curiosidad por el tema, y les pregunte "¿nunca os habéis preguntado por que ese grupo se llama así?". Nadie supo contestarme y comencé a decirles "¿pero que le paso con su oreja?"...

Una vez que tuve captada su atención, comencé a explicarles quien era Van Gogh y a mostrarles sus obras. En un par de días todos sabían quien era Van Gogh, pero me lo escribían mal. En algún caso vi disparates como "Bangot" o, como mucho, "Vangog", pese a que yo lo había escrito correctamente en la pizarra. Todo era inútil, mis alumnos se quejaban de que yo les ponía nombres muy difíciles de aprender. Entonces, pensé de nuevo en recurrir a los conocimientos que sabía que ellos tenían, y les dije: "mirar Van Gogh era holandés como Van Gaal". Inmediatamente lo comprendieron y me dijeron toda una serie de nombres de futbolistas holandeses que yo ni conocía, y les dije: "veis como no es difícill". A partir de entonces rara vez el nombre de Van Gogh aparecía mal escrito.

Aunque este episodio podría entenderse como un hecho aislado, podemos asegurar que no lo es, y que este tipo de situaciones se repiten en la educación secundaria bastante a menudo. Los alumnos están a la última de cualquier novedad en música (para jóvenes), moda, deporte o incluso en temas de cotilleo bastante vulgares. En definitiva, están a la última en todo lo que aparece por la televisión, pero carecen de los conocimientos más básicos sobre arte y están desvinculados de la actualidad cultural. Pero no quiero basarme solo en mi experiencia como profesora, por si este estudio pudiese pecar de excesivamente personal o subjetivo. Para demostrar la realidad de la incidencia del arte en la educación preparé una serie de encuestas para el alumnado de la ESO y el Bachillerato, con el fin de comprobar si existían o no cambios relevantes entre ambas etapas de la Educación Secunsaria, que fueron cumplimentadas en varios centros docentes públicos y privados de la región de Murcia.

Otra cuestión, que pretendo demostrar, es cómo el sistema educativo, incluso los padres - es decir, la sociedad en general-, le da más importancia a las asignaturas en las que se adquieren unos conocimientos lingüísticos o matemáticos que a las artísticas. En palabras de Gardner (1995: 26), "hemos puesto las inteligencias lingüística y lógico-matemática, en sentido figurado, en un pedestal". 


\subsection{Resultados de las encuestas}

A continuación analizaremos el resultado de las cien encuestas realizadas a alumnos de $1^{\circ}, 3^{\circ}$ y $4^{\circ}$ de la ESO y $1^{\circ}$ de Bachillerato.

En estas encuestas se les preguntó, primero, que les preocuparía más suspender Matemáticas o Educación Plástica, Lengua o Educación Plástica, Ciencias Sociales o Educación Plástica. Los resultados fueron estos:

- Al 87\% de los alumnos encuestados le preocupa más suspender las Matemáticas, la Lengua o las Ciencias Sociales que la Educación Plástica.

- Al 3\% también le preocupa más suspender las Matemáticas o la Lengua que Educación Plástica, pero hicieron una excepción con las Ciencias Sociales, anteponiendo la Educación Plástica solo a esta asignatura.

- El 3\% dejó las preguntas en blanco o nulo.

- Por ultimo, solamente el 7\% reconoció tener más preocupación en suspender Educación Plástica que alguna de las asignaturas importantes. En estos casos las respuestas variaban de forma aleatoria: unas veces les preocupaba suspender Educación Plástica más que Matemáticas pero no que Lengua, y otras marcaban Ciencias Sociales y Lengua pero no Matemáticas.

Hay que destacar también que si comparamos la evolución por cursos, es decir, de $1^{\circ}$ de la ESO a Bachillerato, ese $7 \%$ de alumnos que preferían suspender alguna de las asignaturas consideradas más importantes antes que Plástica eran de $1^{\circ} \mathrm{o} 3^{\circ}$ de la ESO, ninguno de $4^{\circ}$ de la ESO o de Bachillerato. Este hecho demuestra que conforme, tienen más edad su preocupación por suspender Educación Plástica disminuye y les preocupan más las asignaturas de Matemáticas, Lengua y Ciencias Sociales.

Cuando se les pregunto a los cien alumnos encuestados qué les preocupaba más a sus padres que suspendieran - Matemáticas o Educación Plástica, Lengua o Educación Plástica, Ciencias Sociales o Educación Plástica一, el resultado fue muy similar:

- E1 90\% de los alumnos piensan que a sus padres les preocupa más que suspendan Matemáticas, Lengua o Ciencias Sociales que Educación Plástica.

- E1 3\% dejo las preguntas en blanco o nulo.

- Un 7\% contesto las preguntas de forma variada. Unas veces decían que les preocupaba más suspender Matemáticas, pero no Lengua y otras veces más Ciencias sociales que Educación Plástica, o al contrario. Es decir, hubo un 7\% de respuestas variables frente a las mayoritarias.

Hasta ahora hemos constatado la preocupación por suspender una u otra asignatura, pero ¿les gusta realmente a los alumnos la Educación Plástica? Para averiguarlo se les formuló una pregunta indirecta: "Imagina que la asignatura de educación Plástica fuera optativa, ¿se la recomendarías a un/a amigo/a?". Estos fueron los resultados:

-El 75\% dijo que sí, frente al 23\% que dijo que no, y un 2\% en blanco o nulo. De lo cual podemos deducir que sí les gusta la asignatura, aunque respondieran que no les preocupa tanto suspenderla como otras.

Seguidamente pasamos, en la encuesta, a comprobar la incidencia del arte actual en su formación y si mantenían algún contacto con la realidad artística de su entorno. 
Primero comprobamos sus conocimientos sobre la actualidad artística. Con este fin les propusimos que escribiesen todos los nombres de pintores y escultores actuales (en activo en los últimos 10 años) que fuesen capaces de recordar, hasta un máximo de 20. Como resultado obtuvimos 17 respuestas correctas y 3 nulas.

Sin embargo, cuando les propusimos que escribiesen todos los nombres de deportistas actuales (en activo en los últimos 10 años) que fuesen capaces de recordar, hasta un máximo de 20, obtuvimos 1.080 respuestas correctas. Es más, algunos escribieron más de 20 pero no fueron computados.

Los resultados son obvios. Además hay otro dato preocupante: cuando hacemos una comparación por cursos el problema no parece solucionarse mucho con la edad, ni con el paso de los adolescentes por nuestras aulas. Así. por ejemplo, de entre 30 alumnos de $1^{\circ}$ de Bachillerato encuestados 25 dejaron en blanco la casilla de los pintores y 29 la de los escultores, mientras que ninguno dejo en blanco la de los deportistas.

También les preguntamos cuando fue la última vez que fueron a ver una exposición de arte y qué fue lo que les impulsó a ello. La mayoría contestó que hacía más de dos años, y un $31 \%$ que no había ido nunca. Por otra parte, la mayoría de los que habían ido fue porque les llevó el centro docente en el que se hallaban inscritos.

\section{Conclusiones}

De lo expuesto hasta ahora podemos concluir que numerosos pedagogos e investigadores valoran las potencialidades del arte como herramienta educativa para fomentar capacidades creativas, intuitivas, manuales, expresivas y visuales. Desde esta posición es pertinente recordar que el concepto de desarrollo humano debe ser un concepto holístico; es decir, que tenga en cuenta todas las capacidades del ser humano sin darles más importancia a unas que a otras. Para colaborar en la consecución de esta meta, la educación a través del arte ofrece un interesante marco educativo en el cual se pueden desarrollar capacidades que quedan relegadas en otros campos, como las citadas anteriormente, además de promover el desarrollo y el autoconocimiento. Por otra parte, la actividad artística entendida como un camino de autorrealización, cuando va acompañada de la autoobservación y de la introspección, puede convertirse en una herramienta de autoconocimiento.

No podemos resumir en estas conclusiones todas las posibilidades que nos brinda el arte como medio formador y trasformador del ser humano, ya que el arte es una herramienta muy versátil y de múltiples usos. No obstante, si podemos afirmar que tras realizar un sondeo en la ESO y el Bachillerato, creemos que se están desaprovechando o infravalorando las potencialidades de esta herramienta.

Los resultados de las encuestas realizadas nos demuestran que, pese a que la asignatura de Educación Plástica resulta atractiva a la mayoría de los alumnos, no constituye una preocupación prioritaria a la hora de aprobar o suspender pues a dicha mayoría le preocupa más suspender Matemáticas, Lengua o Ciencias sociales. Además, los alumnos contestaron básicamente en la misma proporción que a sus padres les preocupaba más un suspenso en las otras asignaturas que en Educación Plástica. Es evidente que la asignatura donde se pueden desarrollar todas las capacidades enumeradas anteriormente está infravalorada en relación con las otras. 
Del mismo modo, el conocimiento de nuestros adolescentes sobre la actualidad artística es mínimo comparado con sus conocimientos en otros aspectos de la actualidad. Este hecho minimiza la incidencia del arte en la educación desaprovechándose así todo el potencial formador del mismo.

Al contrastar las teorías de los defensores de la educación a través del arte con la realidad educativa actual, llegamos a la conclusión de que las tesis de investigadores tan prestigiosos como Gardner caen en saco roto. Por tanto, nos parece obvio sugerir una revisión de algunos aspectos de nuestro sistema educativo con el fin de conseguir un desarrollo humano más completo.

\section{Referencias bibliograficas.}

EISNER, Elliot W. (1998): El ojo ilustrado. Indagación cualitativa y mejora de la práctica educativa. Barcelona, Paidós.

GARDNER, Howard (1994): Educación artística y desarrollo humano. Barcelona, Paidós.

GARDNER, Howard (1995): Inteligencias múltiples, la teoría en la práctica. Barcelona, Paidós.

READ, Herbert (1975): Imagen e idea. La función del arte en el desarrollo de la conciencia humana. México, Fondo de Cultura Económica.

\section{Silvia VIÑAO MANZANERA}

silviavinao@gmail.com

Universidad Católica de Murcia (UCAM) 